\title{
Observation of Bose-Einstein Condensation of Molecules
}

\author{
M.W. Zwierlein, C.A. Stan, C.H. Schunck, S.M.F. Raupach, S. Gupta, Z. Hadzibabic, and W. Ketterle \\ Department of Physics, MIT-Harvard Center for Ultracold Atoms, and Research Laboratory of Electronics, \\ MIT, Cambridge, MA 02139
}

(Dated: November 26, 2003; revised December 5, 2003)

\begin{abstract}
We have observed Bose-Einstein condensation of molecules. When a spin mixture of fermionic ${ }^{6} \mathrm{Li}$ atoms was evaporatively cooled in an optical dipole trap near a Feshbach resonance, the atomic gas was converted into ${ }^{6} \mathrm{Li}_{2}$ molecules. Below $600 \mathrm{nK}$, a Bose-Einstein condensate of up to 900,000 molecules was identified by the sudden onset of a bimodal density distribution. This condensate realizes the limit of tightly bound fermion pairs in the crossover between BCS superfluidity and Bose-Einstein condensation.

PACS numbers: $03.75 . \mathrm{Ss}, 05.30 . \mathrm{Jp}$
\end{abstract}

Over the last few years, many different approaches have been used to cool and trap molecules [1, 2]. One major goal has been the creation of molecular Bose-Einstein condensates which could lead to advances in molecular spectroscopy, studies of collisions, and precision tests of fundamental symmetries.

Recently, a new technique for creating ultracold molecules led to major advances towards molecular BoseEinstein condensation. Molecules were produced from ultracold atoms 3 , 4, 5, 6, 7, 8, 9] near a Feshbach resonance [10], where a molecular state is resonant with the atomic state and molecules can form without heat release. These molecules are highly vibrationally excited and would usually undergo fast decay. However, in the case of fermionic atoms the molecules showed very long lifetimes [7, 8, 9, 11]. This has been attributed to Pauli suppression of the vibrational quenching process which couples a very weakly bound molecular state to much more tightly bound lower lying vibrational states 12 . We have now been able to cool such molecules to BoseEinstein condensation.

This Bose-Einstein condensate represents one extreme of the crossover from Bose-Einstein condensation of tightly bound pairs (molecules) to BCS superfluidity of Cooper pairs, where fermions form delocalized pairs in momentum space [13].

In most of the recent experiments, molecules were formed by sweeping an external magnetic field through the Feshbach resonance, adiabatically converting atoms to molecules [3, 4, 5, 6, 8]. This atom-molecule coupling is a coherent two-body process [14].

In the case of ${ }^{6} \mathrm{Li}$, experimental work indicated [7, 9], and theoretical work predicted [15, 16] that cooling the atoms at constant magnetic field would create an atommolecule mixture in thermal equilibrium. In this case, the atoms and molecules are coupled by three-body recombination [17]. For temperatures lower than the binding energy of the molecular state, an almost pure molecular gas should form, and at even lower temperatures, a molecular Bose-Einstein condensate. This work demonstrates that this surprisingly simple method to create molecular condensates works. The success of this approach depends on a very favorable ratio of collisional rates for formation and decay of molecules which may be unique to ${ }^{6} \mathrm{Li}$.

The goal of molecular BEC was reached in several steps. Using Feshbach resonances, atomic condensates were put into an atom-molecule superposition state [2]. Pure molecular gases made of bosonic atoms were created close to [6] or clearly in [4] the quantum-degenerate regime, but the effective heating time (of about $2 \mathrm{~ms}$ in Ref. [4]) was too short to reach equilibrium. Earlier this month, while this work was in progress, two papers were submitted. Ref. 18 observed a quantum degenerate gas of potassium molecules with an effective lifetime of 5 to $10 \mathrm{~ms}$, sufficiently long to reach equilibrium in two dimensions and to form a non-equilibrium or quasi condensate [19]. Ref. 20] provided indirect evidence for a long-lived condensate of lithium molecules [21]. Here we observe the formation of a condensate by evaporative cooling of a molecular gas close to equilibrium.

The basic scheme of the experiment is similar to our earlier work when we identified two Feshbach resonances in lithium by monitoring the loss of trapped atoms due to three-body recombination as a function of the external magnetic field 22]. This process leads to ultracold molecules in the highest vibrational state below the continuum. However, no attempt was made to detect these molecules because until very recently [7, 9] they were believed to decay rapidly.

Our experimental setup was described in Refs. 22, 23]. After laser cooling and sympathetic cooling with sodium atoms in a magnetic trap, 35 million lithium atoms in the $\left|F, m_{F}\right\rangle=|3 / 2,3 / 2\rangle$ state were transferred into an optical trap formed by a single far detuned laser beam with up to $7 \mathrm{~W}$ of power at $1064 \mathrm{~nm}$. The beam had a $20 \mu \mathrm{m}$ waist and was aligned horizontally along the symmetry axis of the magnetic trap. This generated a $650 \mu \mathrm{K}$ deep trapping potential with $15 \mathrm{kHz}$ radial and $175 \mathrm{~Hz}$ axial trapping frequencies. They were determined with an accuracy of $10 \%$ by exciting dipolar oscillations with an atomic sodium condensate and scaling them to lithium 
atoms using the ratios of polarizabilities and masses.

The ${ }^{6} \mathrm{Li}$ atoms were then transferred to the lowest energy state $|1\rangle$, using an adiabatic frequency sweep around the lithium hyperfine splitting of $228 \mathrm{MHz}$. DC magnetic fields of up to $1025 \mathrm{G}$ could be applied, a range encompassing the $|1\rangle-|2\rangle$ Feshbach resonance 22, 24, 25] where $|2\rangle$ denotes the second lowest hyperfine state of ${ }^{6} \mathrm{Li}$.

Most of our experiments were performed at a magnetic field of $770 \mathrm{G}$. This was below, but still within the width of the broad Feshbach resonance. Here the atomic scattering length is positive corresponding to a stable weakly bound molecular state. Using RF-induced transitions near $80 \mathrm{MHz}$, an equal mixture of states $|1\rangle$ and $|2\rangle$ was prepared with a ratio of temperature $T$ to Fermi temperature $T_{F}$ around 0.3 . The sample was cooled in $350 \mathrm{~ms}$ by ramping down the laser power of the optical trap to typically $10^{-3}$ of the maximum power resulting in a calculated trap depth for unbound atoms of $650 \mathrm{nK}$. The weakly bound molecules have twice the atomic polarizability. They experience the same trap frequencies and twice the trap depth as the lithium atoms. Therefore, we expect mainly atoms to be evaporated.

Atoms and molecules were detected by absorption imaging after ballistic expansion times of 1 to $30 \mathrm{~ms}$. During the time-of-flight, the magnetic field was suddenly switched off, and atoms in both states were imaged simultaneously since the two optical transition frequencies are equal at zero field. Molecules were detected by first dissociating them by sweeping the magnetic field across the Feshbach resonance up to $925 \mathrm{G}$, and then by imaging the resulting atoms at zero field. With the Feshbach sweep, molecules and residual atoms were imaged together. Without it, only the unbound atoms were detected after switching off the magnetic field. We have found that during the initial phase of the evaporative cooling the atomic population dominated. A significant molecule fraction formed around $T \sim 2 \mu \mathrm{K}$, and in the final phase of the cooling, no atoms could be discerned. The absorption images and profiles shown in Figs. 11 and 2 therefore represent purely molecular column densities.

When the laser power of the optical dipole trap was ramped down, the shrinking size of the cloud in absorption imaging signaled lower temperatures. Very abruptly, the smooth distribution changed to a bimodal distribution - the well-known "smoking gun" of Bose-Einstein condensation [26, 27] (Figs. 11 and 2). Due to a slight asymmetry of our trapping potential, the centers of the condensate and of the thermal cloud were shifted.

The phase transition could be identified by plotting the effective area of the cloud vs. laser power $P$ (Fig. 3(a)). At the phase transition, there was an abrupt change in slope whereas the temperature changed smoothly. For a classical gas, the area depends only on temperature and trap frequencies, which vary smoothly with $P$.

Quantitative information on temperature, total atom number, and condensate fraction was obtained by fitting

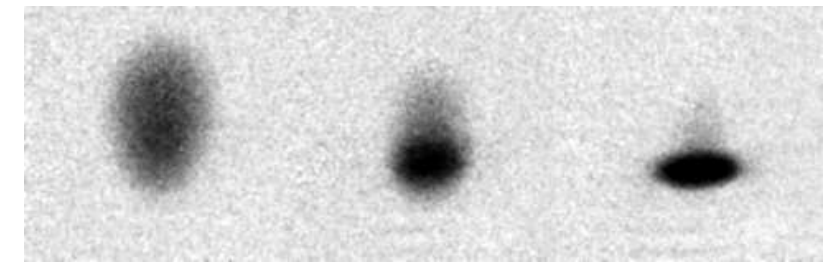

FIG. 1: Observation of Bose-Einstein condensation in a molecular gas. Shown are three single-shot absorption images after $6 \mathrm{~ms}$ of ballistic expansion for progressively lower temperatures (left to right). The appearance of a dark spot marks the onset of BEC. The field of view for each image is $1.4 \mathrm{~mm} \times 1.4 \mathrm{~mm}$. The long axis of the optical dipole trap was vertical in the image.

axial profiles (like in Fig. 2) using a bimodal distribution: a Bose-Einstein distribution for the broad normal component and a Thomas-Fermi distribution for the narrow (condensate) component. Condensates containing up to 900,000 molecules and condensate fractions of up to $75 \%$ were obtained. The onset of BEC was observed at a temperature of $600 \mathrm{nK}$ with $1.4 \times 10^{6}$ molecules. For an ideal gas with this number of molecules, the predicted BEC transition temperature $T_{C}=0.94 \hbar \bar{\omega} N^{1 / 3} / k_{B}$ is $650 \mathrm{nK}$, where $\bar{\omega}$ denotes the geometric mean of the three trapping frequencies. This agreement is fortuitous, given the uncertainty in the trap frequencies at low power [28].

The cool down is characterized in Fig. [3. Axial temperatures were obtained from the spatial extent of the thermal cloud (the size stayed almost constant during the time-of-flight). The axial temperatures are in arbitrary units [28]. Absolute radial temperatures were obtained from the ballistic expansion. All of our temperature measurements are consistent with equilibration in three dimensions.

From the expansion of the largest condensates, we determined the mean field energy $\mu$ to be $300 \pm 100 \mathrm{nK}$. The peak density $n$ of the condensate was obtained from the fit to be $7 \times 10^{13} \mathrm{~cm}^{-3}$. The relation $\mu=4 \pi \hbar^{2} a n / m$, where $m$ is the molecular mass, implies a molecular scattering length of $a=8 \mathrm{~nm}$ with an uncertainty of \pm 60 $\%$. These uncertainties were estimated from the discrepancy of fits done at different times of flight which were not fully consistent. This might reflect asymmetries and anharmonicities of the trapping potential which were not characterized.

It was predicted that the molecular scattering length $a$ is 0.6 times the atomic scattering length $a_{a}$ [12]. At our magnetic field of $770 \mathrm{G}$, the predicted value of $a_{a}$ lies between 140 and $340 \mathrm{~nm}$ depending on the uncertain position of the Feshbach resonance between 810 and $850 \mathrm{G}$ [25]. The discrepancy between the predicted and observed values of $a$ needs further study.

The molecular gas decayed faster than extrapolated from Refs. [7, 9]. Just above $T_{c}$, the thermal cloud had a 


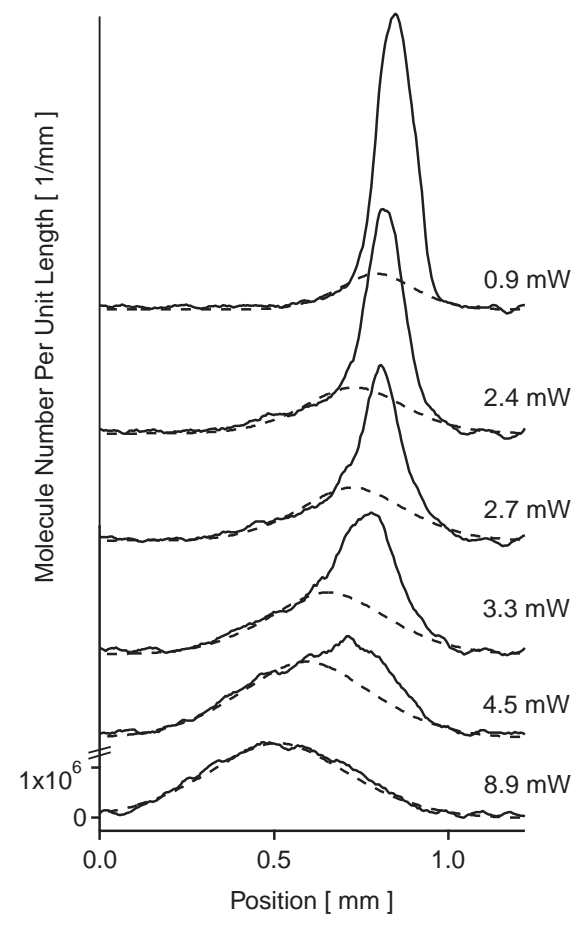

FIG. 2: Bimodality in the density distribution of a molecular gas. Shown are radially averaged profiles through absorption images like those in Fig. 11 as a function of final laser power. The dashed lines are fits to the thermal clouds.

peak density of $1 \times 10^{13} \mathrm{~cm}^{-3}$ and an initial decay time of about $1 \mathrm{~s}$. Condensate numbers decayed to one third after a hold time of $30 \mathrm{~ms}$. Those short lifetimes may reflect leakage or heating in our optical dipole trap at low laser power. In the present experiments, the laser power was not stabilized. The lifetime of the thermal gas is much longer than estimated values of the axial trap period of $100 \mathrm{~ms}$ and of the collision time of $2 \mathrm{~ms}$, which should lead to full equilibrium. Depending on how the condensate grew during the evaporative cooling, its lifetime may have been too short to develop phase-coherence in the axial direction [19.

Using the experimentally determined scattering length, we find that the molecular cloud at $T_{c}$ has a ratio of mean-free path to radius close to 10 and should show only negligible anisotropy during ballistic expansion 19 . Therefore, the onset of strongly anisotropic expansion is a distinguishing feature of the molecular condensate (Fig. 11).

The ${ }^{6} \mathrm{Li}_{2}$ molecules are extremely weakly bound. The molecular binding energy depends on the atomic scattering length $a_{a}$ as $\hbar^{2} / m a_{a}^{2}[29]$. For an assumed $a_{a}=$ $200 \mathrm{~nm}$ the binding energy is $2 \mu \mathrm{K}$. The molecular state which causes the Feshbach resonance is the $X^{1} \Sigma_{g}^{+}, v=$ 38 state. This state is tightly bound, but near the Feshbach resonance it is strongly mixed with the state of the colliding atoms, and the molecular wavefunction is
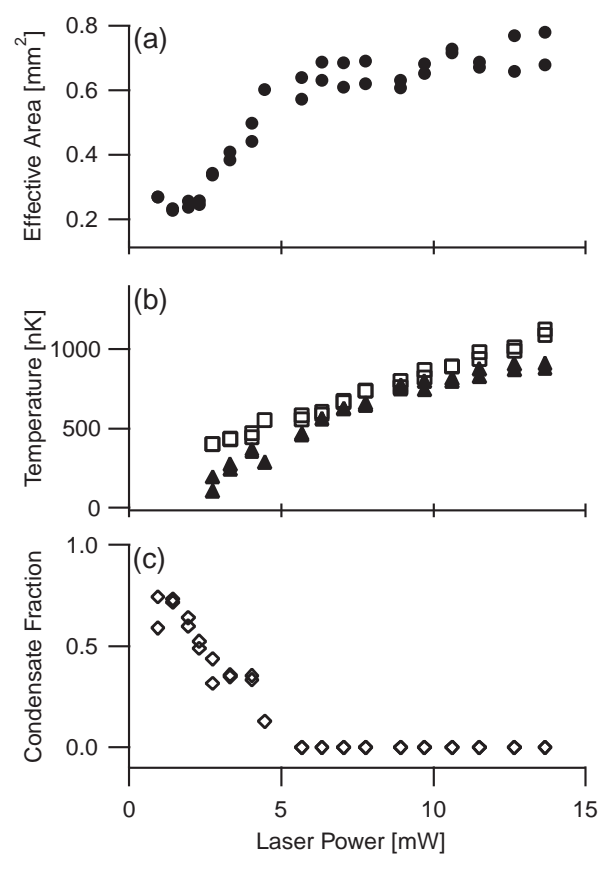

FIG. 3: Characterization of the phase transition. (a) The effective area is the integrated optical density divided by the peak optical density of the absorption images. The "sudden shrinking" of the area coincides with the appearance of a bimodal density distribution and indicates the BEC phase transition. (b) The radial (squares) and axial (triangles) temperatures varied smoothly during the evaporation. The axial temperatures are in arbitrary units. (c) Condensate fraction. Below the critical temperature, the condensate fraction increased from zero to up to $75 \%$.

spread out over an extension of order $a_{a} / 2[29]$.

Direct evidence for the large size of the molecules was obtained by resonant imaging during ballistic expansion at high magnetic fields. At $770 \mathrm{G}$, molecules could be directly imaged using light in resonance with the atoms at the same field. The absorption was comparable to the zero-field absorption signal obtained after dissociating the molecules. This shows that the molecular bond is so weak that the absorption line is shifted from the atomic line by less then a linewidth $\Gamma$. The molecules are expected to absorb most strongly near the outer turning point $R$. The excited state potential is split by the resonant van der Waals interaction $\zeta \hbar \Gamma(\lambda / R)^{3}$ where $\lambda$ is the resonant wavelength divided by $2 \pi$, and $\zeta$ is $\pm 3 / 4, \pm 3 / 2$ for different excited molecular states. The observed absorption signal implies a molecular size $R \geq 100 \mathrm{~nm}$. It is this huge size compared with the much smaller size of the molecule in lower vibrational states which, together with Fermi statistics, inhibits vibrational relaxation and leads to the long lifetimes [12].

Condensates were observed after evaporative cooling at various magnetic fields between 720 and $820 \mathrm{G}$. At 
the lower magnetic fields, the condensate expanded more slowly, consistent with the predictions of a smaller repulsive mean-field energy.

In future work we plan to use the molecular BEC as the starting point for studying the BEC-BCS crossover 13. By ramping up the magnetic field across the Feshbach resonance, the molecules are dissociated into fermionic atoms and the interaction between the atoms changes from repulsive to attractive allowing for the formation of Cooper pairs. Starting with an almost pure condensate and conserving entropy, a Fermi sea should form with temperatures well within the range where BCS type superfluidity has been predicted [30, 31].

In conclusion, we have realized Bose-Einstein condensates of up to 900,000 molecules by evaporative cooling of a spin mixture of fermionic lithium atoms.

Note added in proof: In an optical trap with a slightly enlarged beam waist, we were recently able to hold molecular condensates for up to $400 \mathrm{~ms}$ (or three axial trapping periods) which should result in $3 \mathrm{D}$ equilibration. The $1 / \mathrm{e}$ decay time was about $200 \mathrm{~ms}$.

This work was supported by the NSF, ONR, ARO, and NASA. We thank A. Leanhardt for helpful comments. S. Raupach is grateful to the Dr. Jürgen Ulderup foundation for a fellowship.

[1] J. D. Weinstein, R. DeCarvalho, T. Guillet, B. Friedrich, and J. M. Doyle, Nature 395, 148 (1998); T. Takekoshi, B. M. Patterson, and R. J. Knize, Phys. Rev. Lett. 81, 5105 (1998); R. Wynar, R. S. Freeland, D. J. Han, C. Ryu, and D. J. Heinzen, Science 287, 1016 (2000); H. L. Bethlem, G. Berden, F. M. H. Crompvoets, R. T. Jongma, A. J. A. van Roij, and G. Meijer, Nature 406, 491 (2000); C. Chin, A. J. Kerman, V. Vuletic, and S. Chu, Phys. Rev. Lett. 90, 033201 (2003).

[2] E. A. Donley, N. R. Claussen, S. T. Thompson, and C. E. Wieman, Nature 417, 529 (2002).

[3] C. A. Regal, C. Ticknor, J. L. Bohn, and D. S. Jin, Nature 424, 47 (2003).

[4] K. Xu, T. Mukaiyama, J. R. Abo-Shaeer, J. K. Chin, D. E. Miller, and W. Ketterle, Phys. Rev. Lett. 91, 210402 (2003).

[5] S. Dürr, T. Volz, A. Marte, and G. Rempe, preprint cond-mat/0307440

[6] J. Herbig, T. Kraemer, M. Mark, T. Weber, C. Chin, H.-C. Nägerl, and R. Grimm, Science 301, 1510 (2003).

[7] J. Cubizolles, T. Bourdel, S. J. J. M. F. Kokkelmans, G. V. Shlyapnikov, and C. Salomon, preprint cond-mat/0308018

[8] K. E. Strecker, G. B. Partridge, and R. G. Hulet, Phys. Rev. Lett. 91, 080406 (2003).

[9] S. Jochim, M. Bartenstein, A. Altmeyer, G. Hendl, C. Chin, J. H. Denschlag, and R. Grimm, preprint cond-mat/0308095

[10] W. C. Stwalley, Phys. Rev. Lett. 37, 1628 (1976); E. Tiesinga, B. J. Verhaar, and H. T. C. Stoof, Phys. Rev. A 47, 4114 (1993); S. Inouye, M. R. Andrews, J. Stenger,
H.-J. Miesner, D. M. Stamper-Kurn, and W. Ketterle, Nature 392, 151 (1998); P. Courteille, R. S. Freeland, D. J. Heinzen, F. A. van Abeelen, and B. J. Verhaar, Phys. Rev. Lett. 81, 69 (1998).

[11] C. A. Regal, M. Greiner, and D. S. Jin, preprint cond-mat/0308606

[12] D. S. Petrov, C. Salomon, and G. V. Shlyapnikov, preprint cond-mat/0309010.

[13] M. Randeria, in Bose-Einstein Condensation, edited by A. Griffin, D. Snoke, and S. Stringari (Cambridge University Press, Cambridge, 1995), pp. $355-392$; H. T. C. Stoof, M. Houbiers, C. A. Sackett, and R. G. Hulet, Phys. Rev. Lett. 76, 10 (1996); E. Timmermans, K. Furuya, P. W. Milonni, and A. K. Kerman, Phys. Lett. A 285, 228 (2001); M. Holland, S. J. J. M. F. Kokkelmans, M. L. Chiofalo, and R. Walser, Phys. Rev. Lett. 87, 120406 (2001); Y. Ohashi and A. Griffin, Phys. Rev. Lett. 89, 130402 (2003).

[14] P. D. Drummond, K. V. Kheruntsyan, and H. He, Phys. Rev. Lett. 81, 3055 (1998); P. Tommasini, E. Timmermans, M. Hussein, and A. Kerman, preprint cond-mat/9804015 F. A. van Abeelen and B. J. Verhaar, Phys. Rev. Lett. 83, 1550 (1999); F. H. Mies, E. Tiesinga, and P. S. Julienne, Phys. Rev. A 61, 022721 (2000).

[15] S. J. J. M. F. Kokkelmans, G. V. Shlyapnikov, and C. Salomon, preprint cond-mat/0308384

[16] C. Chin and R. Grimm, preprint cond-mat/0309078

[17] P. O. Fedichev, M. W. Reynolds, and G. V. Shlyapnikov, Phys. Rev. Lett. 77, 2921 (1996); B. D. Esry, C. H. Greene, and H. Suno, Phys. Rev. A 65, 010705 (2001); D. S. Petrov, Phys. Rev. A 67, 010703 (2003).

[18] M. Greiner, C. A. Regal, and D. S. Jin, Nature 426, 537 (2003).

[19] I. Shvarchuck, C. Buggle, D. S. Petrov, K. Dieckmann, M. Zielonkowski, M. Kemmann, T. G. Tiecke, W. v. Klitzing, G. V. Shlyapnikov, and J. T. M. Walraven, Phys. Rev. Lett. 89, 270404 (2002).

[20] S. Jochim, M. Bartenstein, A. Altmeyer, G. Hendl, S. Riedl, C. Chin, J. H. Denschlag, and R. Grimm, Science (to be published).

[21] This work inferred the presence of a condensate from the number of particles in a shallow trap, and the magnetic field dependence of the loss rate consistent with mean field effects. No observation of a phase transition or measurements of equilibration, temperature or condensate fraction were reported.

[22] K. Dieckmann, C. A. Stan, S. Gupta, Z. Hadzibabic, C. H. Schunck, and W. Ketterle, Phys. Rev. Lett. 89, 203201 (2002).

[23] Z. Hadzibabic, S. Gupta, C. A. Stan, C. H. Schunck, M. W. Zwierlein, K. Dieckmann, and W. Ketterle, Phys. Rev. Lett. 91, 160401 (2003).

[24] T. Bourdel, J. Cubizolles, L. Khaykovich, K. M. F. Magalhães, S. J. J. M. F. Kokkelmans, G. V. Shlyapnikov, and C. Salomon, Phys. Rev. Lett. 91, 020402 (2003).

[25] S. Gupta, Z. Hadzibabic, M. W. Zwierlein, C. A. Stan, K. Dieckmann, C. H. Schunck, E. G. M. v. Kempen, B. J. Verhaar, and W. Ketterle, Science 300, 1723 (2003).

[26] M. H. Anderson, J. R. Ensher, M. R. Matthews, C. E. Wieman, and E. A. Cornell, Science 269, 198 (1995).

[27] K. B. Davis, M.-O. Mewes, M. R. Andrews, N. J. van Druten, D. S. Durfee, D. M. Kurn, and W. Ketterle, Phys. Rev. Lett. 75, 3969 (1995). 
[28] The trap frequencies were scaled from measurements done at full power. The axial frequency was affected by small magnetic field curvatures which were negligible at high laser power.

[29] T. Köhler, T. Gasenzer, P. S. Julienne, and K. Burnett, Phys. Rev. Lett. 91, 230401 (2003).
[30] L. D. Carr, G. V. Shlyapnikov, and Y. Castin, preprint cond-mat/0308306

[31] J. N. Milstein, S. J. J. M. F. Kokkelmans, and M. J. Holland, Phys. Rev. A 66, 043604 (2002). 